\title{
A rare novel mutation in TECTA causes autosomal dominant nonsyndromic hearing loss in a Mongolian family
}

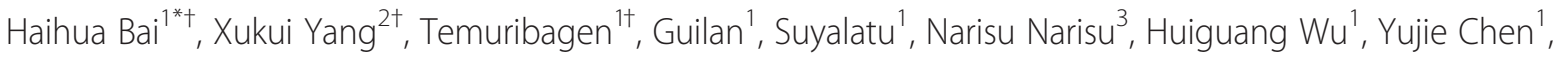
Yangjian Liu ${ }^{4}$ and Qizhu Wu ${ }^{2 *}$

\begin{abstract}
Background: The genetic basis of autosomal dominant nonsyndromic hearing loss is complex. Genetic factors are responsible for approximately 50\% of cases with congenital hearing loss. However, no previous studies have documented the clinical phenotype and genetic basis of autosomal dominant nonsyndromic hearing loss in Mongolians.

Methods: In this study, we performed exon capture sequencing of a Mongolian family with hereditary hearing loss and identified a novel mutation in TECTA gene, which encodes a -tectorin, a major component of the inner ear extracellular matrix that contacts the specialized sensory hair cells.

Results: The novel $\mathrm{G} \rightarrow \mathrm{T}$ missense mutation at nucleotide 6016 results in a substitution of amino acid aspartate at 2006 with tyrosine (Asp2006Tyr) in a highly conserved zona pellucida (ZP) domain of a-tectorin. The mutation is not found in control subjects from the same family with normal hearing and a genotype-phenotype correlation is observed.

Conclusion: A novel missense mutation c.6016 G > T (p.Asp2006Tyr) of TECTA gene is a characteristic TECTA-related mutation which causes autosomal dominant nonsyndromic hearing loss. Our result indicated that mutation in TECTA gene is responsible for the hearing loss in this Mongolian family.
\end{abstract}

Keywords: TECTA gene, Mongolian family, Autosomal dominant nonsyndromic hearing loss

\section{Background}

Hearing loss is one of the most common sensory disorders in humans. Genetic factors are responsible for approximately $50 \%$ of cases with congenital hearing loss $[1,2]$. While $30 \%$ of hereditary hearing loss are syndromic associated with specific signs and medical problems, the other $70 \%$ are nonsyndromic [3]. The heredity of nonsyndromic hearing loss has recessive and dominant modes of inheritance. It is estimated that $80 \%$ of genetic forms of hearing loss is autosomal recessive and the remaining $20 \%$ is autosomal dominant [2]. Autosomal dominant nonsyndromic hearing loss (ADNSHL)

\footnotetext{
*Correspondence: hhbai2010@gmail.com; qizhu_wu@sohu.com

${ }^{\dagger}$ Equal contributors

'Inner Mongolia University for the Nationalities, Tongliao, Inner 028000, Mongolia

${ }^{2}$ BGI-Shenzhen, Shenzhen, Guangdong 518083, China

Full list of author information is available at the end of the article
}

is represented by heterogeneity of genetic and clinical features. To date, about 60 loci associated with ADNSHL have been mapped but only 24 genes have been identified [4].

As one of causative genes of ADNSHL, TECTA mutations have been identified in various types of hearing loss, age of onset, progression and frequency involvement in various populations [5-13]. This gene encodes $\alpha$-tectorin, the major noncollagenous component of tectorial membrane which contacts the outer cochlear hair cells and has an important role in intracochlear sound transmission [14]. The $\alpha$-tectorin is composed of three distinct modules: the entactin G1 domain, the zonadhesin (ZA) domain and the zona pellucida (ZP) domain [14]. Missense and nonsense mutations in the entactin G1 domain and ZP domain are associated with hearing impairment at mid-frequency, whereas mutations in the 
ZA domain primarily affect hearing at the high frequencies $[13,15]$. Phenotypes of hearing loss associated with TECTA can range from mild to severe and have pre or postlingual onset [13].

In this study, we examined the genetic basis of ADSHNL in a Mongolian family with hereditary hearing loss by exon-capture sequencing of known genes associated with hearing loss and identified a novel mutation in TECTA gene. This novel mutation causes the aspartate to tyrosine substitution (Asp2006Tyr) in the highly conserved ZP domain of $\alpha$-tectorin. We further studied the genotypephenotype correlation of mutation in TECTA gene with control subjects from the same family with normal hearing and confirmed that mutation in TECTA gene is responsible for the hearing loss in this Mongolian family.

\section{Methods}

\section{Subjects}

Patients of a Mongolian family suffered severe hearing impairment were diagnosed in the Affiliated Hospital of Inner Mongolia University for the Nationalities. Clinical examination showed that none of these patients has any other associated neurological signs, visual dysfunction or diabetes mellitus. Nineteen members of this Mongolian family participated in the study, of which fourteen showed clinical symptoms of hearing impairment. The study protocol was approved by the Committee on Clinical Investigation at the Inner Mongolia University for the Nationalities University. Informed written consent was obtained from all study participants and the study was in accordance with regulations by the Inner Mongolia University for the Nationalities University.

\section{Audiometric evaluation}

Hearing impairment was evaluated by pure tone audiometry (PTA) in a sound-controlled room at frequencies ranging from 500 to $8000 \mathrm{~Hz}$, according to standard protocols. The severity of hearing loss was defined as follows based on the mean PTA using the thresholds measured at 500, 1000, 2000, and $3000 \mathrm{~Hz}$ : normal hearing, below $20 \mathrm{~dB}$; mild hearing impairment, 21 - $40 \mathrm{~dB}$; moderate hearing impairment, 41 - $70 \mathrm{~dB}$; severe hearing impairment, 71 - $95 \mathrm{~dB}$; and profound hearing impairment, > 95 $\mathrm{dB}$ [16]. The range of hearing loss was described as follows depending on the PTA: low frequency, 125 - 1000 $\mathrm{Hz}$; mid-frequency, $1000-4000 \mathrm{~Hz}$; and high frequency, $4000-8000 \mathrm{~Hz}$.

\section{Target exon capture sequencing}

Approximately $4 \mathrm{~mL}$ of peripheral blood were collected from each study participant and genomic DNA was extracted using the QIAamp DNA Blood Mini Kit (Qiagen, Hilden, Germany). The qualified genomic DNA sample of the affected subject $\mathrm{III}_{21}$ was randomly fragmented by
Covaris (Covaris S2, Massachusetts, USA) into segments ranging from $200 \mathrm{bp}$ to $300 \mathrm{bp}$. These DNA fragments were purified and quantified, and then adapters were ligated to both ends. After amplified by ligation-mediated PCR (LM PCR), adapter-DNA fragments were hybridized to the capture-array of BGI (BGI, Shenzhen, China) to capture all target genes for $72 \mathrm{~h}$. After washing off non-hybridized fragments, captured library which contains the targeted exon sequences of 54 known genes associated with hearing loss was loaded on the Hiseq2000 platform (Illumina, San Diego, USA). Raw image files were processed by Illumina base calling Software 1.7 for base calling with default parameters and the sequences were generated as $90 \mathrm{bp}$ paired-end reads.

After removing adapter sequences and low quality sequence reads from the raw data, the remaining clean reads were mapped and aligned to the human reference genome (NCBI build 37.1/hg19) using Burrows Wheeler Aligner (BWA). SNPs and indels (insertion and deletion) were called by SOAPsnp and GATK (http://www.broadinstitute.org/gsa/wiki/index.php/, The Genome Analysis Toolkit), respectively. All variants of the samples were filtered by databases including dbSNP137 (http://hgdownload. cse.ucsc.edu/goldenPath/hg19/database/snp137.txt.gz), HapMap project (ftp://ftp.ncbi.nlm.nih.gov/hapmap), 1000 Genome Project (ftp://ftp.1000genomes.ebi.ac.uk/vol1/ $\mathrm{ftp}$ ), and an in-house database of BGI. After filtering, variants were further analyzed by SIFT and PolyPhen to predict the effect of mutation on the function of encoding proteins. Only variants that had significant impact on the function of the target protein were further analyzed.

\section{Sanger sequencing}

Candidate variants observed via target exon capture sequencing were screened in other family members who participated the study using conventional capillary sequencing. Briefly, DNA fragments containing variants were PCR amplified from 10-40 ng of genomic DNA using a touch down protocol with primers listed in Table 1. PCR products were visualized on agarose gels, purified with Sephadex columns in accordance with the manufacturers' protocols. Sequence analysis was performed with the Big Dye Terminator Cycle Sequencing Kit and the ABI PRISM 3730 DNA Analyzer (Applied

Table 1 Primers of TECTA and SLC26A4 gene

\begin{tabular}{llc}
\hline Loci & Primer sequence (5'-3') & Size (bp) \\
\hline TECTA-F & GGTCACTTTCAAATGTAAAGG & 443 \\
TECTA-R & CACTGTCCCATCAAAGATGAC & \\
SLC26A4-F & GCTGATATCATGGTTTTCATG & 581 \\
SLC26A4-R & ACACAAATAGGACTATTGAAGG & \\
\hline
\end{tabular}


Biosystems). Sequence traces were analyzed using the Sequencher 4.7 program (Gene Codes Corporation).

\section{Results}

\section{ADSHNL diagnosis and clinical study}

We ascertained a Mongolian family with autosomal dominant nonsyndromic hearing loss. The pedigree comprises four generations with 19 affected family members ( 9 males and 10 females), 14 of whom are willing to participate in this study (Figure 1). There was no evidence of any other cause of hearing impairment, except for one patient who had undergone stapes replacing surgery for unilateral otosclerosis. This patient was included in this study but only audiometric data from her other non-surgical ear were used. The first symptoms of hearing impairment were self-reported at ages ranging from 1 to 69 years. No vestibular symptoms were reported. Otoscopy was normal in all subjects except the abovementioned patient. Pure tone audiograms (PTA) were symmetric and often displayed a so-called cookie-bite shape, which indicates that the survey frequencies are predominantly affected (Figure 2). The highest threshold was most often found at $1000 \mathrm{~Hz}$ and followed by 2000 Hz. The PTA was usually within the range of 40-120 dB and apparently independent of age. By contrast, the PTA from control subjects with normal hearing was in the range of $0-40 \mathrm{~dB}$ (Figure 2).

\section{Identification of candidate mutation}

To understand the genetic basis underlying the hearing loss in this Mongolian family, we took the candidate gene approach to screen 54 known hearing loss associated genes with exon capture followed by deep sequencing of the DNA sample from the patient III21. We then analyzed the SNPs and indels mutation from sequencing reads. The bioinformatic analysis identified four missense variants (p.Arg371Gly, p.Val932Ala, p.Ser1724Asn and p.Asp2006Tyr) and four synonymous variants (p.Ala495Ala, p.Ile752Ile, p.Tyr935Tyr, p.Ser1878Ser) in coding region of TECTA (Table 2), which encodes $\alpha$-tectorin, a major component of the inner ear extracellular matrix that contacts the specialized sensory hair cells. One missense mutation (p.Cys282Gly) in SLC26A4 was also called in the aforementioned analyzed strategy. No other mutation was identified in the rest of known hearing loss associated genes, including ACTG1, CCDC50, COCH, COL11A2, DFNA5, DIAPH1, and EYA4 et al. for this patient sample.

The sequencing coverage of every exon of TECTA was up to $99 \%$, and the depth of each exon was close to the average median-depth across 23 exons, indicating sequencing in our study is deep enough for identifying all possible variants (Figure 3B). This suggests the abovementioned variants are the possible causative mutations impairing the hearing of this Mongolian family. To narrow down the candidate variants of TECTA, we then filtered these variants with four databases (dbSNP, HapMap, 1000 project and in-house database of $\mathrm{BGI}$ ) with a minor allele frequency (MAF) of 0.005. Only p.Asp2006Tyr was left after filtering, suggesting a novel variant in TECTA.

\section{Genotype-phenotype correlation study}

The TECTA gene is known as a causative gene for DFNA8/DFNA12 and DFNB21 hearing loss in humans, and $S L C 26 A 4$ is related to autosomal recessive hearing loss. The missense mutations p.Asp2006Tyr of $\alpha$ tectorin and p.Cys282Gly of SLC26A4 are heterozygous, which fits the autosomal dominant inheritance model. These two mutations have not been reported previously based on the public databases. The p.Asp2006Tyr of $\alpha$-tectorin and p.Cys282Gly of SLC26A4 were predicted to be pathogenic mutations by SIFT and PolyPhen. Thus, p.Asp2006Tyr of $\alpha$-tectorin and p.Cys282Gly of SLC26A4 were considered as candidate causative mutations of hearing loss in the studied Mongolian family. Therefore, we further screened these mutations in other affected subjects using Sanger sequencing for a genotype phenotype correlation analysis with primers listed in Table 1.

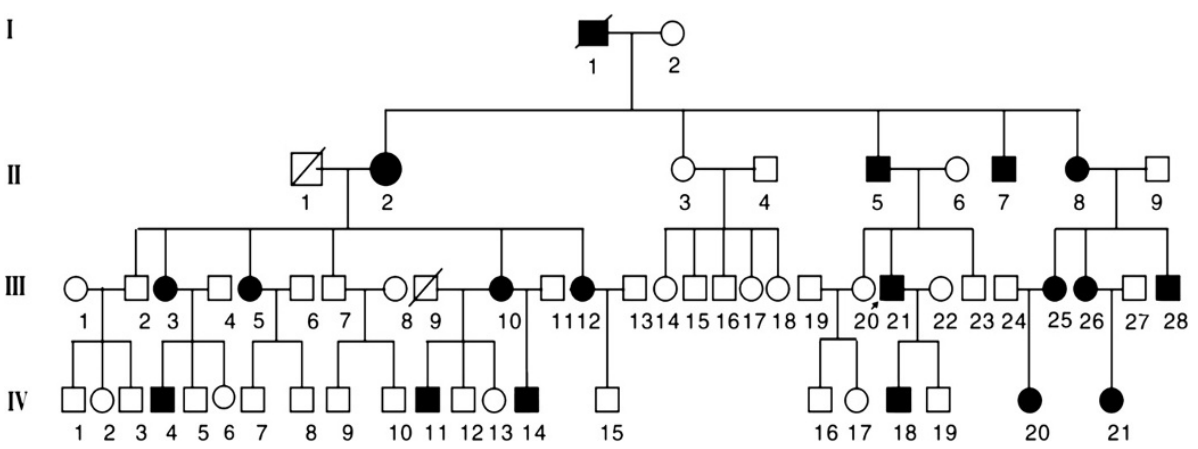

Figure 1 Pedigree of the Mongolian family. Black and white symbols indicate the affected and the unaffected subjects, respectively. 


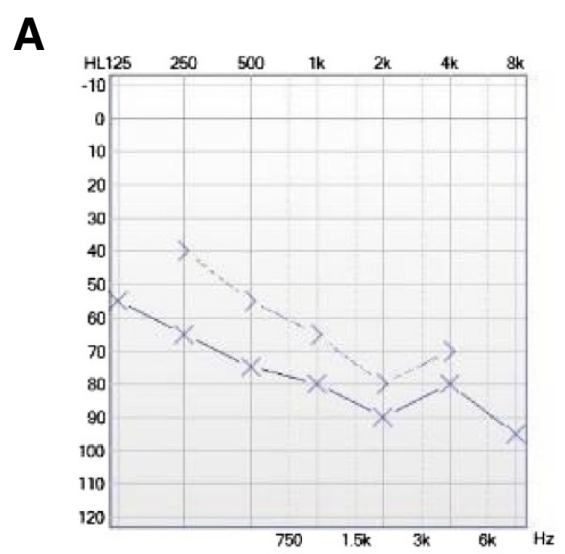

II-5 (64 yrs old)

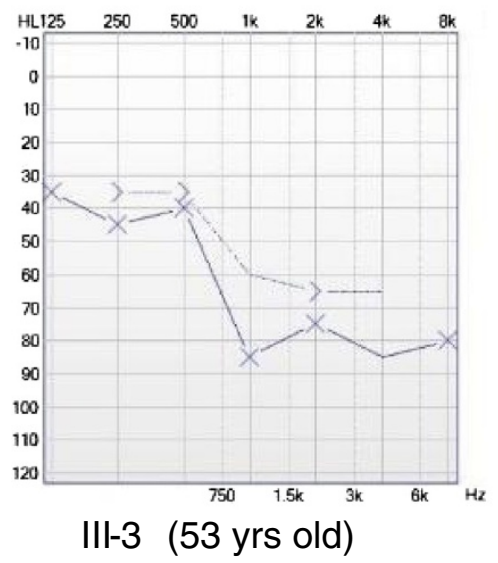

B

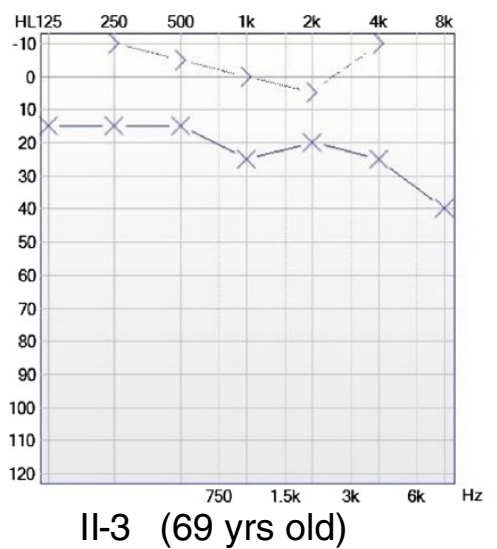

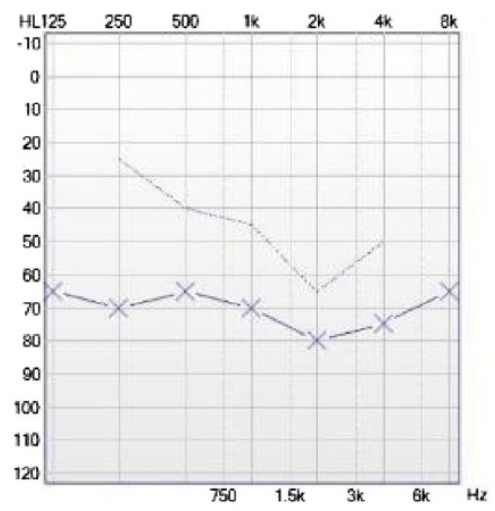

II-7 (60 yrs old)
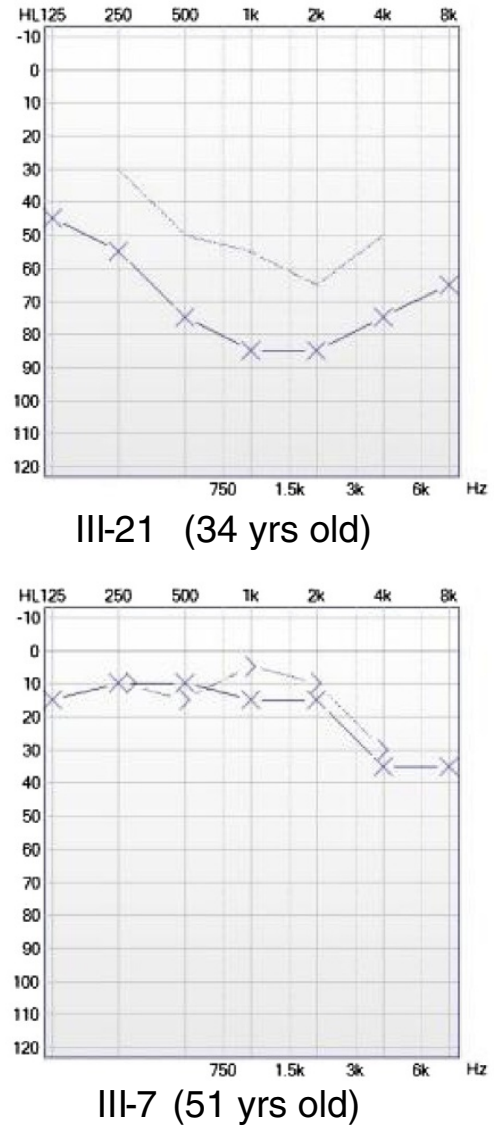

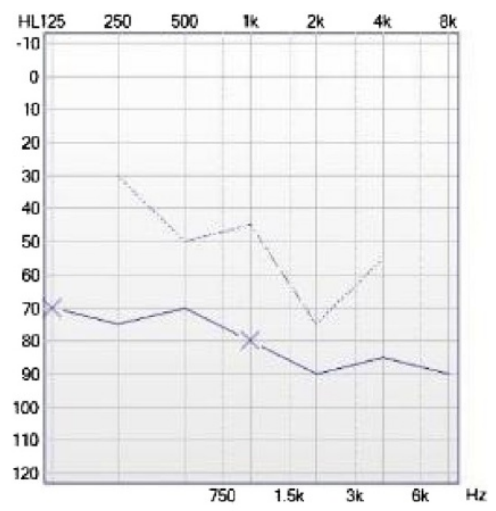

II-8 (55 yrs old)
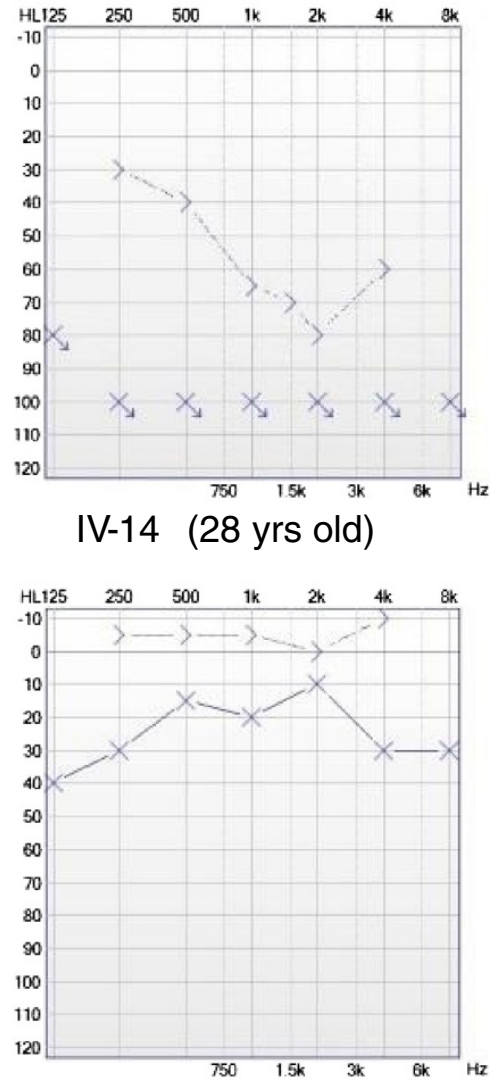

III-11 (43 yrs old)

Figure 2 Pure tone audiometry recording of representative members in the Mongolian family. A. audiograms of six different patients in three generations (only audiograms of left ear is shown). $X$ : frenquency; $Y$ : intensity (dB). B. audiograms of the left ear of three different control subjects in two generations. The age of each subject at the time of tested was indicated.

Sequencing of three patients and three control subjects in the family showed that c.844 T $>$ G (p.Cys282Gly) in SLC26A4 is also present in controls, ruling out the association of p.Cys282Gly in SLC26A4 with hearing loss in the studied Mongolian family. We then confirmed the presence of the novel missense mutations c.6016 G>T (p.Asp2006Tyr) of the TECTA gene in exon 20 of the rest of all 13 participated subjects with hereditary hearing loss (Figure 4B). The affected members in this family (II-2, III-3, III-5, and III-10) were heterozygous for the mutation, but not detected in the II-1. This is consistent with a complete segregation of the variant allele with the phenotype status in this family (Figures 1 and $4 \mathrm{~B}$ ). This mutation is 
Table 2 Mutations identified by exon-capture sequencing

\begin{tabular}{lccccc}
\hline Variants & Rs_ID & MAF-dbSNP & MAF-HapMap & MAF-1K project & MAF-in-house \\
\hline p.Arg371Gly & rs612969 & 0.5 & 0.394 & 0.4432 & 0.3892 \\
p.Ala495Ala & rs536069 & 0.629 & 0.533 & 0.6007 & 0.4627 \\
p.lle752lle & rs10502247 & 0.449 & 0.474 & 0.402 & 0.4988 \\
p.Val932Ala & rs520805 & - & 0.387 & 0.3526 & 0.3843 \\
p.Tyr935Tyr & rs586473 & 0.588 & 0.504 & 0.5696 & 0.4663 \\
p.Ser1724Asn & rs526433 & - & 0.993 & 0.9936 & 0.9964 \\
p.Ser1878Ser & rs2155369 & - & 0.314 & 0.2244 & 0.4181 \\
p.Asp2006Tyr & novel & - & - & 0 & 0 \\
\hline
\end{tabular}

located in the ZP domain of $\alpha$-tectorin which is highly conserved across species (Figure 4C). We further sequenced DNAs of all the normal control subjects of the family, plus screened additional 201 healthy individuals from a random Mongolian population sample that we have deep sequencing data. We did not find the presence of the above-mentioned $\mathrm{p}$. Asp2006Tyr mutation in the TECTA gene (data not shown), demonstrating the genotype-phenotype correlation of the mutation in TECTA gene with the hearing loss in the Mongolian family.

\section{Discussion}

TECTA encodes $\alpha$-tectorin, the major component of extracellular matrix contacting cochlear hair cells and transmitting sounds [14]. Mutations in $\alpha$-tectorin have been detected in ADNSHL in various populations across different ages of onset and progressions [5-13], accounting for $\sim 4 \%$ of all cases [13]. Interestingly, previous studies showed that mutations affecting three different domains (entactin G1 domain, ZA domain, and ZP) present clinically different phenotypes. Missense mutations on the ZA domain are normally affecting hearing at the high frequencies, whereas mutations on entactin G1 domain and ZP domain are linked to mid-frequency hearing impairment $[13,15]$. ADNSHL patients with mutations in $\alpha$-tectorin display various severity and onset of hearing loss [13].

In this study, we did exon-capture followed by nextgeneration sequencing to screen 54 known hearing-loss associated genes for mutations in a Mongolian family with hereditary hearing loss. We identified four missense variants (p.Arg371Gly, p.Val932Ala, p.Ser1724Asn and p.Asp2006Tyr) and four synonymous variants (p.Ala495Ala, p.Ile752Ile, p.Tyr935Tyr, p.Ser1878Ser) in coding regions of TECTA. Among these, a novel variant, p.Asp2006Tyr was found to be implicated as the pathogenic missense mutation causing hearing loss. All TECTA mutations described to date have shown significant genotype-phenotype correlation with hearing loss [5-13]. In our study, the novel heterozygous mutation
p.Asp2006Tyr on TECTA is only present in affected members of the family and is not found in any of the control subjects in the family plus a fairly large population sample of the same ethic group. Consistent to previous reports that mutations on the $\mathrm{ZP}$ domains are associated with mid-frequency hearing loss, the hearing of affected subjects was predominantly affected at the frequency between $1000 \mathrm{~Hz}$ and $2000 \mathrm{~Hz}$. The clinical phenotype of hearing loss in this family displays early onset (as early as 1 year old). However, no correlation between age and severity of hearing impairment has been observed.

Several recently developed mouse lines with human mutations on Tecta provide extremely useful information for understanding the mechanism of hearing loss in the affected families [17-19]. Mice with missense mutation in the ZP domain of Tecta (Tecta ${ }^{\mathrm{Y} 1870 \mathrm{C} /+}$, Tecta $^{\mathrm{L} 1820 \mathrm{~F}, \mathrm{G} 18724 \mathrm{D} /+}$ and Tecta ${ }^{\mathrm{C} 1837 \mathrm{G} /+}$ ) display hearing loss in mild-frequency range, phenocopying the clinical observation with affected patients $[17,19]$. By contrast, the hearing impairment in mice carrying missense mutation in the ZA domain of Tecta (Tecta ${ }^{\mathrm{C} 1509 \mathrm{G} /+}$ and Tecta $^{\mathrm{C} 1619 \mathrm{~S} /+}$ ) is more subtle and slightly different from what had been reported in patients $[18,19]$. While the Tecta ${ }^{\mathrm{C} 1509 \mathrm{G} /+}$ mouse had increased reverse transduction [18], the Tecta ${ }^{\mathrm{C} 1619 \mathrm{~S} /+}$ mouse was surprisingly found to have a stable rather than progressive deterioration hearing phenotype [19]. The authors suggested the genetic traits of the mouse lines could be a possible explanation for the different deafness phenotype in mouse and human caused by the same mutation. Likewise, the age-independency of hearing loss in the large Mongolian family under this study is probably dues to the slight difference in their individual genetic background. A knockin mouse line with Tecta $^{\mathrm{D} 1619 \mathrm{Y} /+}$ will be very useful to investigate how the point mutation identified in current study affects the onset of deafness under various mouse genetic backgrounds.

This novel variation p.Asp2006Tyr in the ZP domain was a substitution of aspartate with a hydrophilic side chain to tyrosine with a hydrophilic-neutral chain. Previously, various mutations in the ZP domain (C1837G, $\mathrm{R} 2021 \mathrm{H}$, and $\mathrm{Y} 1870 \mathrm{C}$ ) of TECTA had been shown 


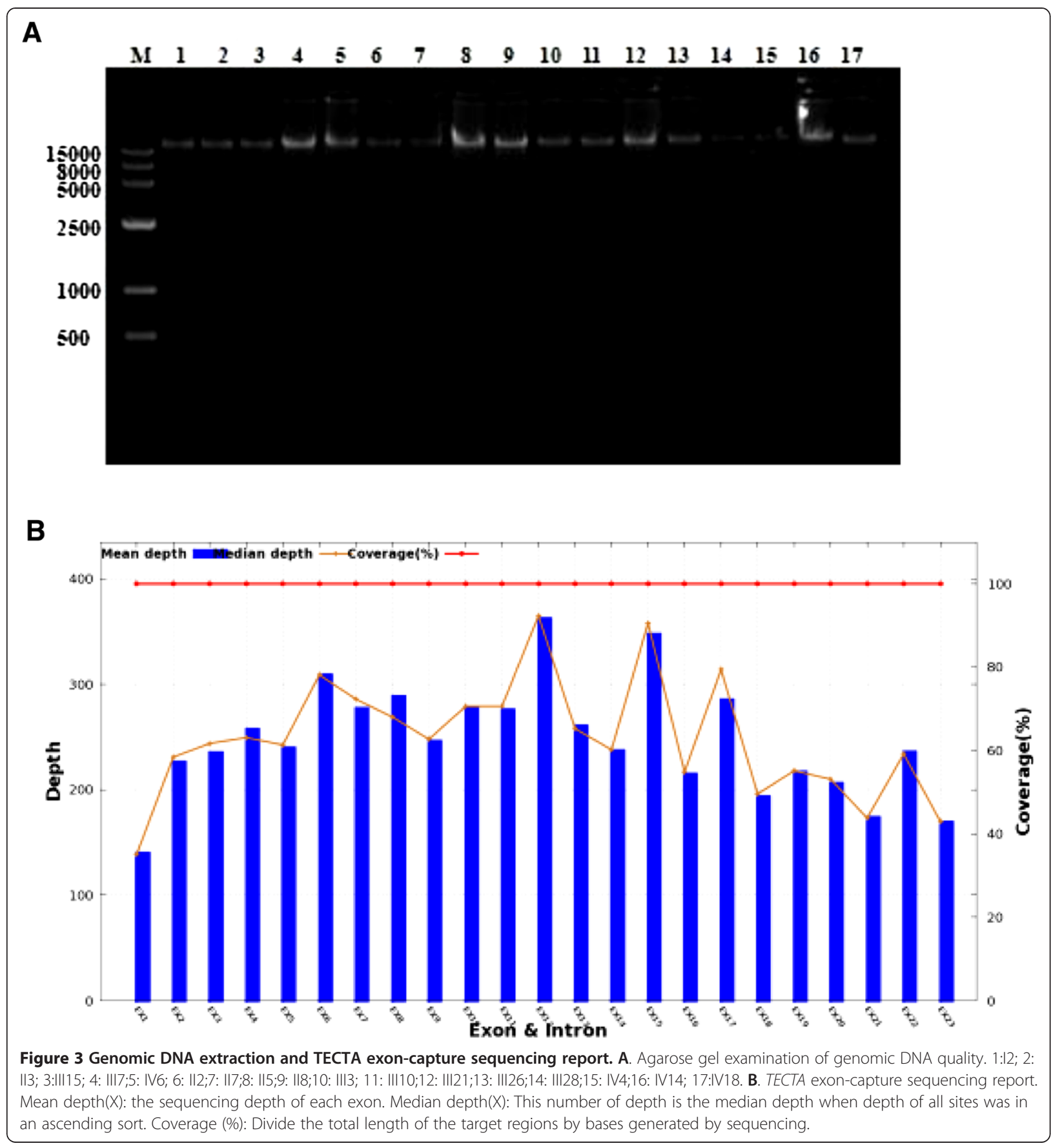

within the cytoplasm, suggesting the defect for the mutants to be secreted outside of the cell properly [20]. It seems likely that the p.Asp2006Tyr mutation in the ZP domain from this study might also cause similar defects in the secretion of $\alpha$-tectorin. However, it is of note that the secretion defect of mutations in the ZP domain was drawn from truncated constructs of TECTA without $\mathrm{N}$-terminal secretion peptide signal. Further cellular studies with full-length constructs are necessary to elucidate the exact mechanism of $\alpha$ tectorin's role in ADNSHL.

\section{Conclusion}

This study documents a novel missense mutations c.6016 $\mathrm{G}>\mathrm{T}$ (p.Asp2006Tyr) of the TECTA gene that causes autosomal dominant nonsyndromic hearing loss in a Mongolian family. This expands the mutation spectrum of TECTA gene in nonsyndromic hearing loss. Our study 


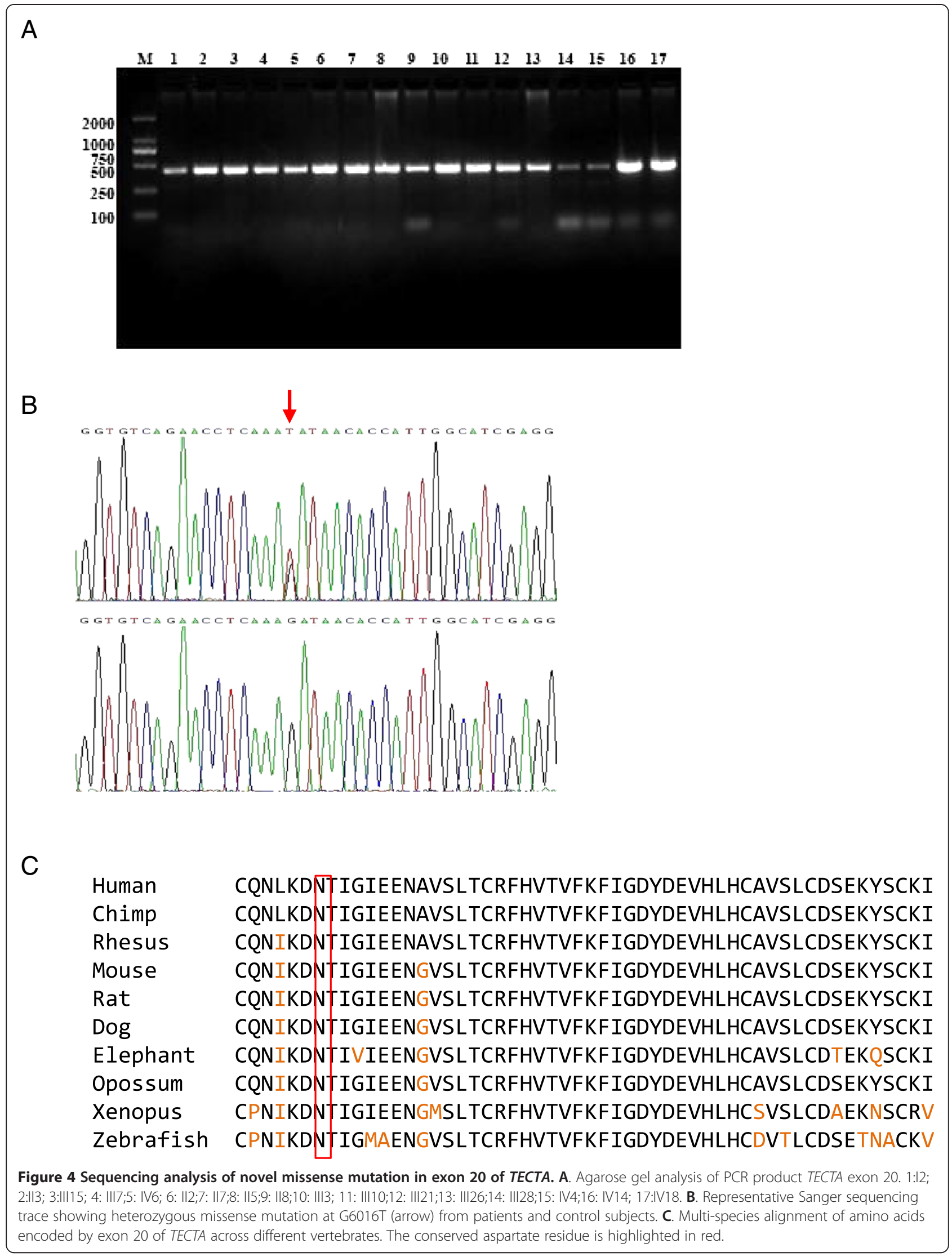


also exemplifies the application of deep sequencing in identifying disease causative mutations from a pedigree.

\section{Competing interests}

The authors declare that they have no competing interests with respect to the present article.

\section{Authors' contributions}

HB designed the study and family recruitment, performed experiments, analyzed and interpreted the data, drafted the manuscript and obtained funding. QW designed the study, conducted clinical diagnoses and obtained funding. XY selected and designed the targets capture, performed experiments, analyzed and interpreted the data. T conceived of the study concept, drafted the manuscript, and analyzed and interpreted the data. NN supervised the study design. YL analyzed and interpreted the data. All other authors provided technical assistance, and all authors read and approved the final manuscript.

\section{Acknowledgements}

We thank each member of the family for their generous participation in this study. This study was supported by the National Natural Science Foundation of China (81160101,81060098).

\section{Author details}

${ }^{1}$ Inner Mongolia University for the Nationalities, Tongliao, Inner 028000, Mongolia. ${ }^{2} \mathrm{BGI}-S h e n z h e n$, Shenzhen, Guangdong 518083, China. ${ }^{3} \mathrm{Genome}$ Technology Branch, National Human Genome Research Institute, National Institutes of Health, Bethesda, MD, USA. ${ }^{4}$ Department of Developmental Biology, Washington University School of Medicine, St. Louis, MO 63110, USA.

Received: 10 November 2013 Accepted: 12 March 2014

Published: 19 March 2014

\section{References}

1. Friedman TB, Griffith AJ: Human nonsyndromic sensorineural deafness. Annu Rev Genomics Hum Genet 2003, 4:341-402.

2. Morton CC, Nance WE: Newborn hearing screening-a silent revolution. N Engl J Med 2006, 354(20):2151-2164.

3. Smith RJH, Shearer AE, Hildebrand MS, Van Camp G: Deafness and hereditary hearing loss overview. In GeneReviews. Edited by Pagon RA, Adam MP, Bird TD, Dolan CR, Fong CT, Stephens K. University of Washington, Seattle; 1993-2014. [http://www.ncbi.nlm.nih.gov/books/ NBK1434/]

4. Hereditary hearing loss homepage. [http://hereditaryhearingloss.org]

5. Iwasaki S, Harada D, Usami S, Nagura M, Takeshita T, Hoshino T: Association of clinical features with mutation of TECTA in a family with autosomal dominant hearing loss. Arch Otolaryngol Head Neck Surg 2002, 128(8):913-917.

6. Verhoeven K, Van Laer L, Kirschhofer K, Legan PK, Hughes DC, Schatteman I, Verstreken M, Van Hauwe P, Coucke P, Chen A, Smith RJ, Somers T, Offeciers FE, Van de Heyning P, Richardson GP, Wachtler F, Kimberling WJ, Willems PJ, Govaerts PJ, Van Camp G: Mutations in the human alpha-tectorin gene cause autosomal dominant non-syndromic hearing impairment. Nat Genet 1998, 19(1):60-62.

7. Pfister M, Thiele H, Van Camp G, Fransen E, Apaydin F, Aydin O, Leistenschneider $\mathrm{P}$, Devoto M, Zenner HP, Blin N, Nürnberg P, Ozkarakas H, Kupka S: A genotype-phenotype correlation with gender-effect for hearing impairment caused by TECTA mutations. Cell Physiol Biochem 2004, 14(4-6):369-376.

8. Balciuniene J, Dahl N, Jalonen P, Verhoeven K, Van Camp G, Borg E, Pettersson $U$, Jazin EE: Alpha-tectorin involvement in hearing disabilities: one gene-two phenotypes. Hum Genet 1999, 105(3):211-216.

9. Alloisio N, Morle L, Bozon M, Godet J, Verhoeven K, Van Camp G, Plauchu H, Muller P, Collet L, Lina-Granade G: Mutation in the zonadhesin-like domain of alpha-tectorin associated with autosomal dominant non-syndromic hearing loss. Eur J Hum Genet 1999, 7(2):255-258.

10. Hughes DC, Legan PK, Steel KP, Richardson GP: Mapping of the alphatectorin gene (TECTA) to mouse chromosome 9 and human chromosome 11: a candidate for human autosomal dominant nonsyndromic deafness. Genomics 1998, 48(1):46-51.
11. Plantinga RF, de Brouwer $A P$, Huygen $P L$, Kunst HP, Kremer $H$, Cremers $C W$ : A novel TECTA mutation in a Dutch DFNA8/12 family confirms genotype-phenotype correlation. J Assoc Res Otolaryngol 2006, 7(2):173-181.

12. Moreno-Pelayo MA, del Castillo I, Villamar M, Romero L, Hernandez-Calvin FJ, Herraiz C, Barbera R, Navas C, Moreno F: A cysteine substitution in the zona pellucida domain of alpha-tectorin results in autosomal dominant, postlingual, progressive, mid frequency hearing loss in a Spanish family. $J$ Med Genet 2001, 38(5):E13.

13. Hildebrand MS, Morin M, Meyer NC, Mayo F, Modamio-Hoybjor S, Mencia A, Olavarrieta L, Morales-Angulo C, Nishimura CJ, Workman H, DeLuca AP, del Castillo I, Taylor KR, Tompkins B, Goodman CW, Schrauwen I, Wesemael MV, Lachlan K, Shearer AE, Braun TA, Huygen PL, Kremer H, Van Camp G, Moreno F, Casavant TL, Smith RJ, Moreno-Pelayo MA: DFNA8/12 caused by TECTA mutations is the most identified subtype of nonsyndromic autosomal dominant hearing loss. Hum Mutat 2011, 32(7):25-834.

14. Legan PK, Lukashkina VA, Goodyear RJ, Kossi M, Russell IJ, Richardson GP A targeted deletion in alpha-tectorin reveals that the tectorial membrane is required for the gain and timing of cochlear feedback. Neuron 2000, 28(1):273-285.

15. Plantinga RF, Cremers CW, Huygen PL, Kunst HP, Bosman AJ: Audiological evaluation of affected members from a Dutch DFNA8/12 (TECTA) family. J Assoc Res Otolaryngol 2007, 8(1):1-7.

16. Lee KY, Choi SY, Bae JW, Kim S, Chung KW, Drayna D, Kim UK, Lee SH: Molecular analysis of the GJB2, GJB6 and SLC26A4 genes in Korean deafness patients. Int J Pediatr Otorhinolaryngol 2008, 72(9):1301-1309.

17. Legan PK, Lukashkina VA, Goodyear RJ, Lukashkin AN, Verhoeven K, Van Camp G, Russell IJ, Richardson GP: A deafness mutation isolates a second role for the tectorial membrane in hearing. Nat Neurosci 2005, 8(8):1035-1042.

18. Xia A, Gao SS, Yuan T, Osborn A, Bress A, Pfister M, Maricich SM, Pereira FA, Oghalai JS: Deficient forward transduction and enhanced reverse transduction in the alpha tectorin C1509G human hearing loss mutation. Dis Model Mech 2010, 3(3-4):209-223.

19. Legan PK, Goodyear RJ, Morin M, Mencia A, Pollard H, Olavarrieta L, Korchagina J, Modamio-Hoybjor S, Mayo F, Moreno F, Richardson GP: Three deaf mice: mouse models for TECTA-based human hereditary deafness reveal domain-specific structural phenotypes in the tectorial membrane. Hum Mol Genet 2014. Epub ahead of print.

20. Moteki H, Nishio SY, Hashimoto S, Takumi Y, Iwasaki S, Takeichi N, Fukuda S, Usami S: TECTA mutations in Japanese with mid-frequency hearing loss affected by zona pellucida domain protein secretion. J Hum Genet 2012, 57(9):587-592.

\section{doi:10.1186/1471-2350-15-34}

Cite this article as: Bai et al:: A rare novel mutation in TECTA causes autosomal dominant nonsyndromic hearing loss in a Mongolian family. BMC Medical Genetics 2014 15:34.

\section{Submit your next manuscript to BioMed Central and take full advantage of:}

- Convenient online submission

- Thorough peer review

- No space constraints or color figure charges

- Immediate publication on acceptance

- Inclusion in PubMed, CAS, Scopus and Google Scholar

- Research which is freely available for redistribution 